\title{
In Vivo Bioluminescence Imaging of Pancreatic Cancer Xenografts in NOG Mice
}

\author{
Hisashi Yoshimura, Yoko Matsuda, Zenya Naito and Toshiyuki Ishiwata* \\ Departments of Pathology and Integrative Oncological Pathology, Nippon Medical School, Tokyo, Japan
}

\begin{abstract}
Implantation of human tumors in immunodeficient mice and in vivo bioluminescence imaging are powerful tools for cancer research. In the present study, we established a novel cell line, PANC-luc5, from PANC-1 pancreatic carcinoma cells transfected with the luciferase gene. We confirmed the usefulness of this cell line by transplanting it into three mouse strains with different immunodeficiency statuses; BALB/cA Jcl-nu/nu (Nude), Crlj:SHO-Prkdcscid $\mathrm{Hr}^{h r}$ (SHO), and NOD/Shi-scid, IL-2 $\gamma^{\text {null }}$ (NOG) mice. NOG mice were also inoculated with PANC-luc5 cells in either the tail vein or abdominal cavity, and monitored with in vivo bioluminescence imaging. Our results show that PANC-luc5 tumors orthotopically transplanted into NOG mice grew steadily and progressed to metastases, but those in Nude and SHO mice remained their original size with no metastasis throughout the experimental period. In the orthotopic and experimental metastasis models, in vivo bioluminescence imaging was used successfully to visualize tumor growth and metastasis of PANC-Iuc5 cells in NOG mice. In conclusion, in vivo bioluminescence imaging using orthotopic, intravenous, and intraperitoneal implantation of PANC-luc5 cells into NOG mice can be used to study the mechanisms of metastasis and to develop anti-pancreatic cancer drugs.
\end{abstract}

Keywords: In vivo bioluminescence imaging; NOG mice; Nude mice; SHO mice; Pancreatic cancer

\section{Introduction}

Implantation of human tumors into immunodeficient mice has provided valuable information about tumor growth and metastasis, and aided in the development of efficient anticancer agents [1,2]. Nude mice, which lack mature $\mathrm{T}$ cells due to congenital thymic hypoplasia, are commonly used for such experiments. Recent efforts to improve the implantation success rate have produced novel mouse strains with more severe immunodeficiency, including Crlj:SHO-Prkd $c^{\text {scid }} H r^{h r}$ (SCID Hairless Outbred: SHO) and NOD/Shi-scid, IL- $2 \gamma^{\text {null }}$ (NOG) mice [2]. SHO mice are a strain of hairless mice derived by crossing the Crlj:Prkds $c^{s i d}$ and Crlj:SKH1-Hr $r^{h r}$ stocks. These mice lack both T and B cells and thereby has an immunodeficiency status similar to that of the SCID mouse. NOG mice are a novel strain of severely immunodeficient mice that were established by backcrossing C57BL/6J $-\gamma \mathrm{c}^{\text {null }}$ mice with NOD/Shi-scid mice [2]. NOG mice have inherited the $\mathrm{T}$ and $\mathrm{B}$ cell deficiency of SCID mice, which carry a Prkdc gene mutation, as well as the macrophage and complement depression of non-obese diabetic (NOD) mice. Additionally, an interleukin-2R $\gamma$ (IL-2R $\gamma$ ) allelic mutation causes this strain to have defective natural killer (NK) and dendritic cells. These combined immunodeficiencies markedly improve the success rate of heterotopic transplantation.

In NOG mice, human hematopoietic stem cells can engraft at a high rate and differentiate into multiple cell lineages [2]. These humanized NOG mice can be efficiently infected with human blood-borne pathogens such as human immunodeficiency virus type 1 (HIV-1) [3]. In addition, functional endometrium or ovary can be regenerated by transplantation of singly-dispersed cells or fragments of human organs into NOG mice $[4,5]$. This strain also shows high engraftment rates for human tumors. Various types of human hematopoietic tumors such as acute myeloid leukemia [6], multiple myeloma [7], and Hodgkin's lymphoma [8] have been reported to efficiently engraft in NOG mice. Furthermore, engraftment of human non-hematopoietic solid tumors (such as epithelial and mesenchymal tumors) can be used to establish in vivo tumor tissue lines that retain the characteristics of the original tumors [1]. These findings suggest that NOG mice are good animal models for understanding the pathogenesis of solid and non-solid tumors; however, there is a limitation in that the normal immune response against cancer inherent in humans is not reflected in the immunodeficient mouse model.

Compared with subcutaneous implantation, orthotopic implantation-in which human pancreatic carcinoma cells are grafted to the murine pancreas- is considered to more closely mimic the biological processes of tumors within the human body [9]. Tumor cell injections into the mouse tail veil and abdominal cavity have been used to study vascular metastasis and peritoneal dissemination, respectively $[10,11]$. However, these orthotopic and experimental metastasis models usually require sacrificing the animals to evaluate the tumor growth and metastasis. Acquiring longitudinal data related to the development of implanted tumors requires sacrificing a large number of mice, which is inconsistent with animal welfare and very expensive. These limitations can be alleviated by recent advances in in vivo imaging systems for small animals, including ultrasound, Positron Emission Tomography (PET), computed tomography, Magnetic Resonance Imaging (MRI), and fluorescence and bioluminescence imaging [12]. Bioluminescence imaging is highly sensitive and relatively inexpensive, and can be used to monitor and quantify the luciferase-gene-transfected cells in a living animal over time [13-16].

In the present study, we used PANC-1 cells to establish a novel pancreatic carcinoma cell line that stably expresses the luciferase gene. We additionally confirmed that these cells could be used with in

*Corresponding author: Toshiyuki Ishiwata, Director of Molecular Pathology Group, Departments of Pathology and Integrative Oncological Pathology, Nippon Medical School, 1-1-5 Sendagi, Bunkyo-ku, Tokyo 113-8602, Japan, Tel: +81-33822-2131 ext. 5232; Fax: +81-3-5814-6274; E-mail: ishiwata@nms.ac.jp

Received May 06, 2013; Accepted June 07, 2013; Published June 17, 2013

Citation: Yoshimura H, Matsuda Y, Naito Z, Ishiwata T (2013) In Vivo Bioluminescence Imaging of Pancreatic Cancer Xenografts in NOG Mice. J Carcinogene Mutagene S9: 003. doi:10.4172/2157-2518.S9-003

Copyright: (c) 2013 Yoshimura H, et al. This is an open-access article distributed under the terms of the Creative Commons Attribution License, which permits unrestricted use, distribution, and reproduction in any medium, provided the original author and source are credited. 
vivo bioluminescence imaging in various types of immunodeficiency mice-particularly super-immunodeficient NOG mice-for pancreatic cancer research.

\section{Materials and Methods}

\section{Cell culture}

PANC-1 cells were obtained from the Cell Resource Center for Biomedical Research; Institute of Development, Aging, and Cancer; Tohoku University (Sendai, Japan). These cells were grown in RPMI 1640 medium containing $10 \%$ fetal bovine serum (FBS) at $37^{\circ} \mathrm{C}$ under a humidified $5 \% \mathrm{CO}_{2}$ atmosphere.

\section{Establishment of a cell line stably expressing the luciferase} gene

The pGL4.50[luc2/CMV/Hygro] vector encoding the luciferase reporter gene luc2 (originating from the North American firefly, Photinus pyralis) was purchased from Promega KK (Tokyo, Japan). PANC- 1 cells $\left(2 \times 10^{5}\right.$ cells) were plated in $35-\mathrm{mm}$ culture dishes and transfected with $5 \mu \mathrm{g}$ of plasmid vector using FuGene HD transfection reagent (Roche Applied Science, Indianapolis, IN, USA). The cells were cultured in $2 \mathrm{ml}$ RPMI 1640 medium containing 10\% FBS and 2mg/ $\mathrm{ml}$ hygromycin. The growing cells were collected and 100 cells were replated in 100-mm culture dishes then independent colonies were isolated by ring cloning.

To select the cell clone with the highest luciferase gene expression, PANC-1 parental cells and 41 clones of PANC- 1 cells transfected with luciferase vector were plated at $1 \times 10^{5}$ cells per well of a 96-well plate. After 24 hours, $0.5 \mathrm{mM}$ luciferin (Beetle Luciferin, Promega KK) was added to each well, and the bioluminescence from each well was estimated with a luminescence image analyzer (ImageQuant LAS4000, GE Health Care Japan Corporation, Tokyo, Japan).

To confirm whether the luminescence intensity correlated with the number of cells, we plated a 2 -fold dilution series of luciferaseexpressing PANC-luc5 cell clones (from 8000 to 31 cells) in 96-well plates in duplicate. After adding $0.5 \mathrm{mM}$ luciferin, the plates were imaged for 60 seconds using IVIS Spectrum version 3.2 (Caliper Life Sciences, Hopkinton, MA). Furthermore, we plated 10, 100, 1000, and 10,000 PANC-luc5 cells in 30-mm culture dishes, and prepared a dish filled with only culture medium as a negative control. After luciferin treatment, images were taken of each dish and the light emission intensity was quantified as Total Flux (photons/second).

\section{Orthotopic implantation}

We suspended $5 \times 10^{6}$ PANC-luc5 cells in $100 \mu \mathrm{l}$ of PhosphateBuffered Saline (PBS), and this suspension was subcutaneously injected into the flanks on both sides of 6 -week-old male BALB/cA Jcl-nu/nu (Nude) mice. After 4 weeks, the mice were imaged by IVIS to confirm emission from the tumors that developed. Then, the animals were sacrificed by ether inhalation, and the tumors were resected from the subcutis and cut into small fragments ( 2 -mm squares). These fragments were sutured to the surface of the pancreatic tails of 6-week-old male Nude $(n=2)$, SHO $(n=2)$, and NOG $(n=2)$ mice under inhalational anesthesia with isoflurane (Table 1).
Using IVIS, images of the mice were taken every week for up to 8 weeks. For stable luciferase expression, mice were imaged within 5 minutes of post-intraperitoneal injection of $100 \mathrm{mg} / \mathrm{kg}$ luciferin $(200$ $\mu \mathrm{l}$ of $10 \mathrm{mg} / \mathrm{ml})$.

\section{Intravenous and intraperitoneal implantation}

Five million Panc-luc5 cells were suspended in $100 \mu \mathrm{PBS}$, and injected into the tail vein of a 6-week-old NOG mouse. Another NOG mouse had $5 \times 10^{6}$ Panc-luc5 cells suspended in $100 \mu \mathrm{l}$ PBS injected into its abdominal cavity. These mice were imaged by IVIS 6 hours after cell injection then once weekly for up to 6 weeks thereafter.

\section{Ex vivo procedure and histopathology of internal organs}

At the end of the experimental period, the mice were dissected after intraperitoneal luciferin administration, and images of the internal organs were taken by IVIS. To histologically confirm the presence of metastasis, the organs were fixed in $10 \%$ neutral buffered formalin, embedded in paraffin wax, and cut into $4-\mu \mathrm{m}$-thick tissue sections. The serial tissue sections were stained with hematoxylin and eosin, autoclaved in citrate buffer ( $\mathrm{pH}$ 6.0) for $15 \mathrm{~min}$, and immunostained using the Histofine Mouse Stain Kit (NICHIREI, Tokyo, Japan) with a mouse monoclonal antibody against HLA class I (A, B, C) (clone EMR8-5, 1:1000, Hokudo, Sapporo, Japan). They were then evaluated under light microscopy. Animal experiments were carried out in accordance with the institutional animal care guidelines of the Nippon Medical School Animal Ethical Committee.

\section{Results}

\section{Generation of human pancreatic carcinoma cells that stably} express luciferase

Forty-one clones were established from PANC-1 cells transfected with the luciferase vector. Luminescence intensities were measured for these clones, and the PANC-luc5 clone showed the highest emission level (Figure 1A arrow). In IVIS images, the wells seeded with serial dilutions of PANC-luc5 cells varied in color from red to violet-blue in accordance with decreasing cell numbers (Figure 1B, left). There was a strong correlation between Total Flux and the number of PANCluc5 cells (Figure 1B, right). The mean Total Flux was approximately 300 photons/second per cell, and no bioluminescence was observed in the negative control with only medium (data not shown). Obvious luminescence signals were observed from the tumors that originated from PANC-luc5 cells injected into the subcutis of the flanks on both sides of the nude mice (Figure 1C).

\section{Imaging of the pancreatic cancer orthotopic implantation model in Nude, SHO, and NOG mice}

In all Nude and SHO mice that had a PANC-luc5 tumor fraction implanted into the pancreas, the amount of luminescence at the implantation site remained unchanged over 8 weeks (Figure 2A, left and center panels). No luminescent signals were observed outside of the implanted site in these mice. On the other hand, in NOG mice the luminescent area of the implanted tumor expanded beyond the implantation site as soon as during the first week of observation

\begin{tabular}{|c|c|c|c|c|c|c|}
\hline \multicolumn{2}{|c|}{ Mouse strain } & Hair & T lymphocytes & B lymphocytes & Natural killer cells & Macrophages, Dendritic cells, Complement \\
\hline BALB/cA Jcl-nu/nu & Nude & - & - & + & + \\
\hline Crlj:SHO-Prkdc & Scid Hri & SHO & - & - & - & + \\
\hline NOD/Shi-scid, IL-2y & NOG & + & - & - & + \\
\hline
\end{tabular}

Table 1: Characteristics of immunodeficiency mice. 
Citation: Yoshimura H, Matsuda Y, Naito Z, Ishiwata T (2013) In Vivo Bioluminescence Imaging of Pancreatic Cancer Xenografts in NOG Mice. J Carcinogene Mutagene S9: 003. doi:10.4172/2157-2518.S9-003

A

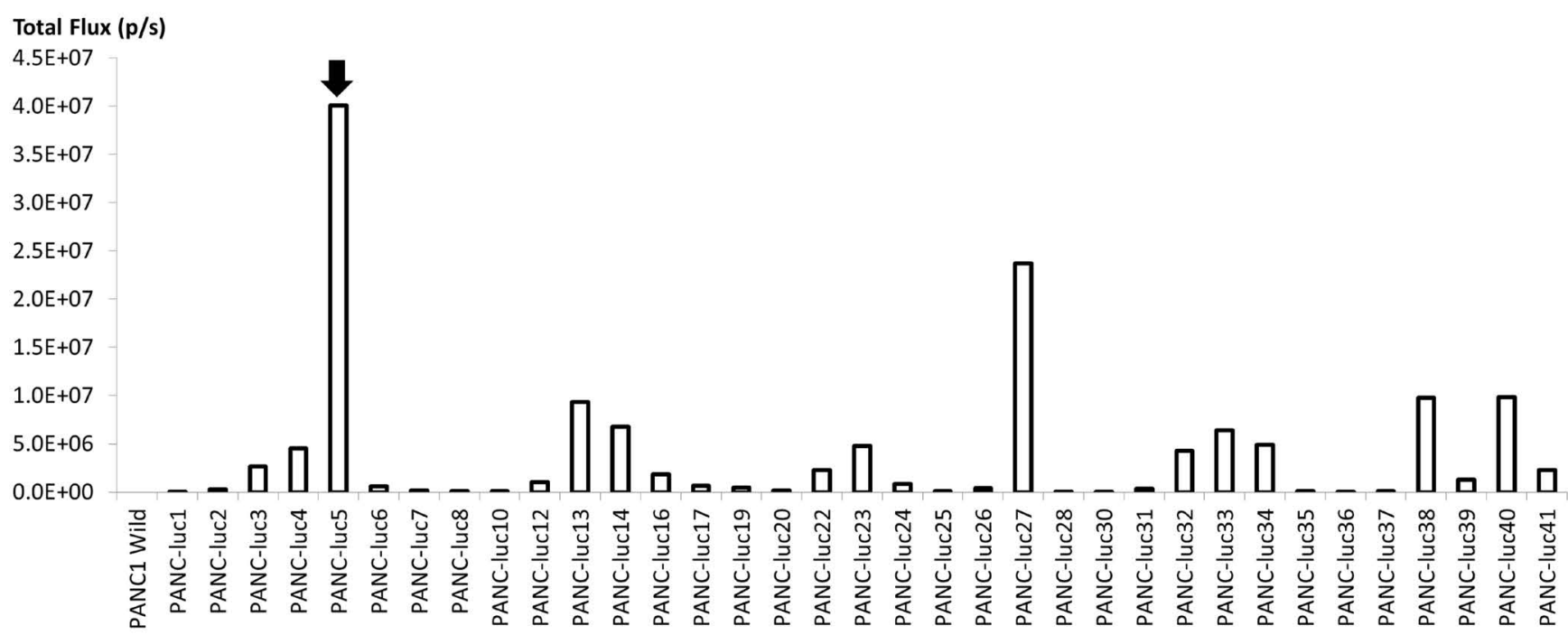

B
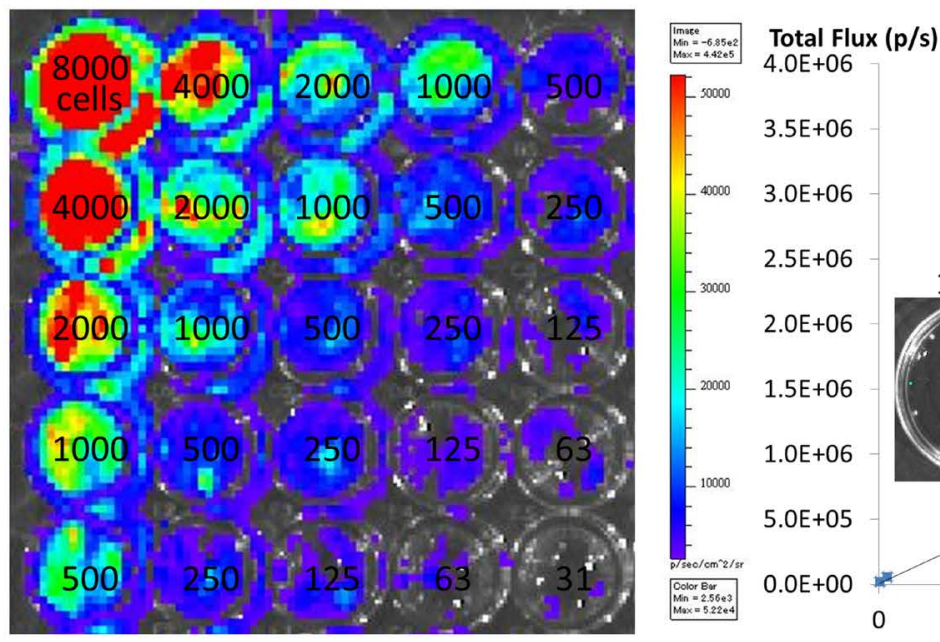

\section{$\mathrm{p} / \mathrm{s})$}

.

$1.5 \mathrm{E}+06$

$1.0 \mathrm{E}+06$

$5.0 \mathrm{E}+05$

$0.0 \mathrm{E}+00$
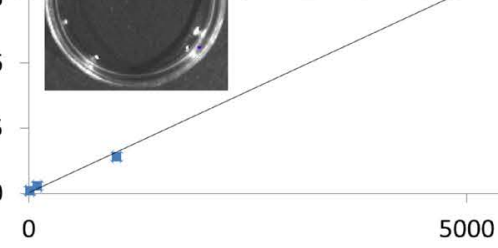

C

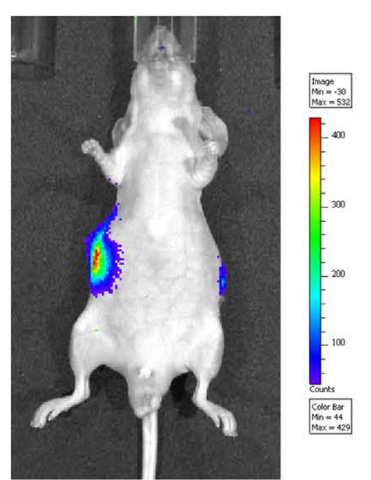

Figure 1: (A) Among 41 clones established from luciferase-transfected PANC-1 cells, PANC-luc5 cells showed the highest emission level (arrow). (B) A bioluminescence image shows that wells seeded with serially diluted PANC-luc5 cells varied in color from red to violet-blue in accordance with decreasing cell numbers (left). There was a strong correlation between Total Flux (photons/second) and the number of PANC-luc5 cells (right). (C) Obvious luminescence signals from subcutaneous tumors developed from PANC-luc5 cells injected into both sides of the lateral region in nude mice. 


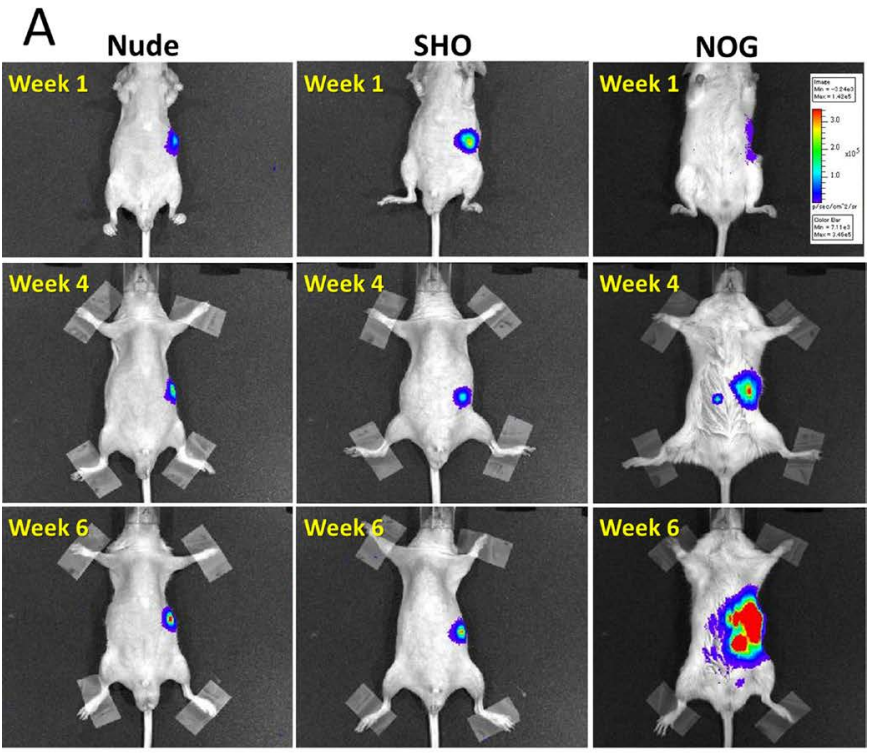

B

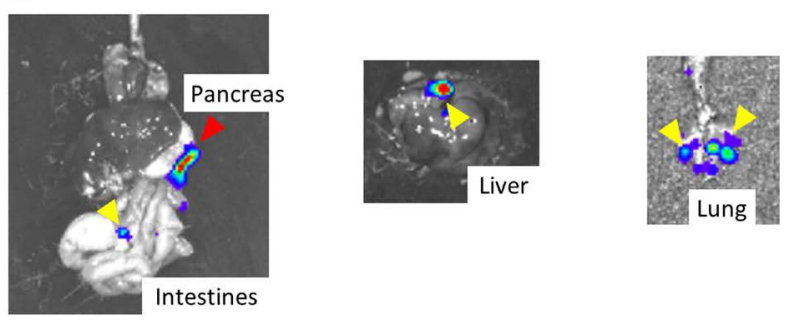

C

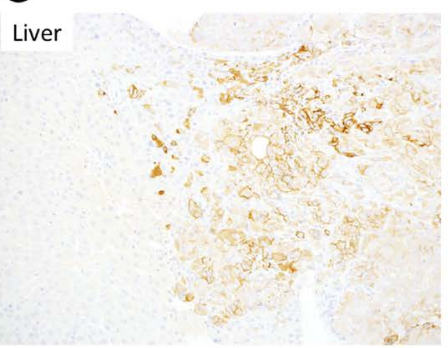

Figure 2: (A) In vivo imaging of an orthotopic implantation model of pancreatic cancer in Nude (left), SHO (center), and NOG (right) mice. In Nude and SHO mice, the size of the luminescence from tumors implanted in the pancreas remained unchanged during the experimental period. On the other hand, the luminescent signal in NOG mice expanded over the week. (B) Ex vivo imaging of internal organs of the NOG mouse showed bioluminescence signals in the pancreas (left; red arrowhead), intestines (left; yellow arrowhead), liver (center; yellow arrowhead), and lungs (right; yellow arrowheads). (C) The immunostaining of HLA class I-A,B,C clearly highlighted the nest of human cancer cells in the liver of the NOG mouse. Original magnification, $\times 200$.

(Figure 2A, right upper panel). Furthermore, during the second week of observation, less intense luminescent signals were observed in areas away from the implantation site, indicating distant metastases (Figure $2 \mathrm{~A}$, right middle and lower panels).

Besides the implanted sites, there were no macroscopically visible metastases in any of the mice during autopsy at week 8 . However, ex vivo imaging of the internal organs excised from NOG mice showed bioluminescence signals in the pancreas (Figure 2B, left panel, red arrowhead), liver (Figure 2B, center panel, yellow arrowhead), lungs
(Figure 2B, right panel, yellow arrowheads), and serous surface of the intestines (Figure 2B, left panel, yellow arrowhead). Staining the organ sections with an anti-HLA class $\mathrm{I}(\mathrm{A}, \mathrm{B}, \mathrm{C})$ antibody that recognizes human, but not mouse cells clearly highlighted nests of human cancer cells in the liver (Figure 2C) and other organs of NOG mice. Ex vivo imaging and histological examination revealed no evidence of metastasis in Nude or SHO mice (data not shown).

\section{Imaging of vascular metastasis and peritoneal dissemination models of pancreatic cancer in NOG mice}

Six hours after intravenous injection of PANC-luc5 cells into the NOG mouse, most of the bioluminescence was localized in the chest (lung regions; Figure 3A, red arrowheads). The intensities were reduced one week later. At weeks 2 and 3 , bioluminescent signals began to increase in the chest again and also appeared in the upper right abdomen (liver region; Figure 3A, yellow arrowheads). From week 4 to week 6 , signals in the upper right abdominal area steadily increased. In the autopsy at the end of the experimental period, metastatic lesions were macroscopically visible in the liver and lung, with the most severe lesions in the liver. Ex vivo imaging of the excised liver (Figure 3B, yellow arrowheads) and lungs (Figure 3B, red arrowhead) showed marked light emission. Less intense light signals were also detected in the heart, stomach, and kidneys. The presence of human cancer cell metastatic foci in the lungs (Figure 3C) and other organs were confirmed in the sections immunostained with anti-HLA class I (A, B, C) antibody.

After intraperitoneal inoculation of the NOG mouse with PANCluc5 cells, the abdominal bioluminescent signals gradually expanded throughout the experimental period (Figure 4A). Ex vivo imaging confirmed bioluminescent signals in the liver, gastrointestinal tract, and kidneys (Figure 4B). HLA class I (A, B, C) immunostaining of tissue sections revealed the presence of human cancer cell nests on the serous surface of the intestines (Figure 4C), liver, and perirenal fat capsule.

\section{Discussion}

In this study, we generated the new cell line, PANC-luc5, from PANC-1 cells stably transfected with the luciferase gene. PANC-1 cells are one of the most commonly used human pancreatic ductal adenocarcinoma cell lines. In vitro bioluminescence imaging of culture dishes with serially-diluted PANC-luc5 cells demonstrated a clear correlation between light emission intensity and the number of cells. PANC-luc5 cells showed tumorigenicity in nude mice, and the tumors that developed produced intense light emission, proving the usefulness of this cell line for bioluminescent imaging experiments. Other researchers have also constructed luciferase-expressing cell lines derived from PANC-1 or other pancreatic carcinoma cell lines and used them for in vivo bioluminescent imaging [17-20].

We studied tumor formation in three strains of immunodeficient mice (Nude, SHO, and NOG) to determine which strain was best suited for PANC-luc5 cell transplantation (Table 1). SHO mice are a recently developed mouse strain and have not yet been widely used in oncological research. The immunodeficiency level of this strain is comparable to that of SCID mice, which lack functional $\mathrm{T}$ and $\mathrm{B}$ lymphocytes, but not NK cells; however, its nude skin makes in vivo imaging easy to perform. NOG mice are another recently developed mouse strain. These mice have multifunctional defects in T, B, NK, macrophage, and dendritic cell functions and complement activity.

We found that PANC-luc5 tumors orthotopically transplanted 
Citation: Yoshimura H, Matsuda Y, Naito Z, Ishiwata T (2013) In Vivo Bioluminescence Imaging of Pancreatic Cancer Xenografts in NOG Mice. J Carcinogene Mutagene S9: 003. doi:10.4172/2157-2518.S9-003

A
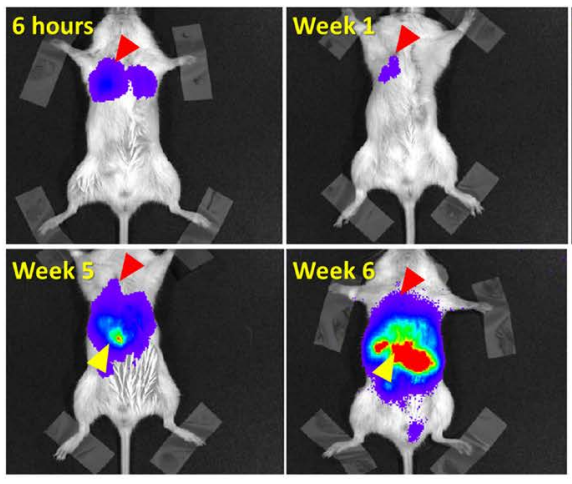

B

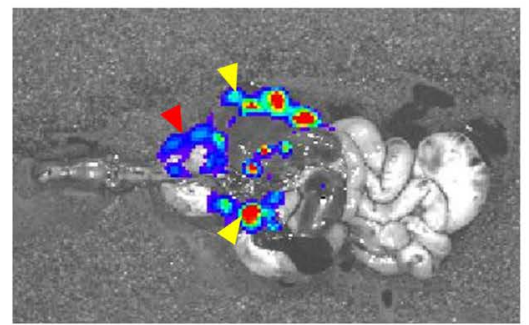

C

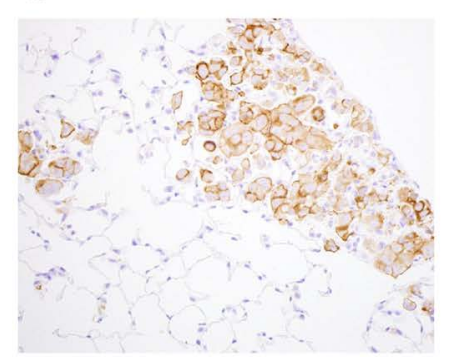

Figure 3: (A) In vivo imaging of the vascular metastasis model of pancreatic cancer in the NOG mouse showed bioluminescence localized in the lung regions (red arrowheads) after 6 hours. The intensity was reduced at 1 week, but then increased again at the lung regions and also appeared at the liver region at 3 weeks (yellow arrowheads). (B) Ex vivo imaging of excised internal organs showed marked light emission from the liver (yellow arrowheads) and lungs (red arrowhead). (C) Metastatic foci of human cancer cells in the lungs immunostained with anti-HLA class I-A,B,C antibody. Original magnification, $\times 400$.

into NOG mice grew steadily and progressed to metastases, but these phenomena were not observed in Nude and SHO mice. This study was performed in a limited number of mice; therefore, an accurate assessment of the differences between strains of mice should be performed based on higher numbers of animals. However, this finding is consistent with previous studies that have compared NOG and other immunodeficient mice. Machida et al. [21] compared the susceptibilities of NOG, SCID, and Nude mice to tumor formation following subcutaneous inoculation with HeLa S3 human cervical cancer cells. Six out of ten NOG mice, but no SCID or Nude mice, developed subcutaneous tumors after inoculation with $1 \times 10^{3}$ cancer cells. Furthermore, even a mere $10^{1} \mathrm{HeLa}$ S3 cells led to tumor formation in five out of ten NOG mice. Ikoma et al. [22] reported liver and lung metastases in NOG mice, but not in NOD/SCID mice, after intravenous inoculation with melanoma cells. Suemizu et al. [23] also compared liver metastasis formation after portal vein injection of human pancreatic carcinoma cells into NOG and NOD/SCID mice. When inoculated with only $1 \times 10^{2}$ PANC- 1 cells, metastasis was observed in three out of eight NOG mice. In contrast, none of the ten NOD/SCID mice developed liver metastasis, even when inoculated with $1 \times 10^{4}$ PANC- 1 cells. The high susceptibility of NOG mice to tumor xenografts is assumed to be largely attributable to their NK cell deficiency. The NK cell state of immunodeficient mice is known to be a key factor affecting the success or failure of xenograft transplantation [24]. It has also been suggested that unknown properties of the strain may further contribute to the high rate of xenograft uptake by NOG mice [2]. Overall, NOG mice are a superior recipient for human tumors, and serve as an excellent model for studies of tumor metastasis.

In the orthotopic and experimental metastasis models using PANC-luc5 cells, in vivo bioluminescence imaging clearly visualized the behavior of the transplanted tumors within the living animals. In the vascular metastasis model, the autopsy at the end of the experimental

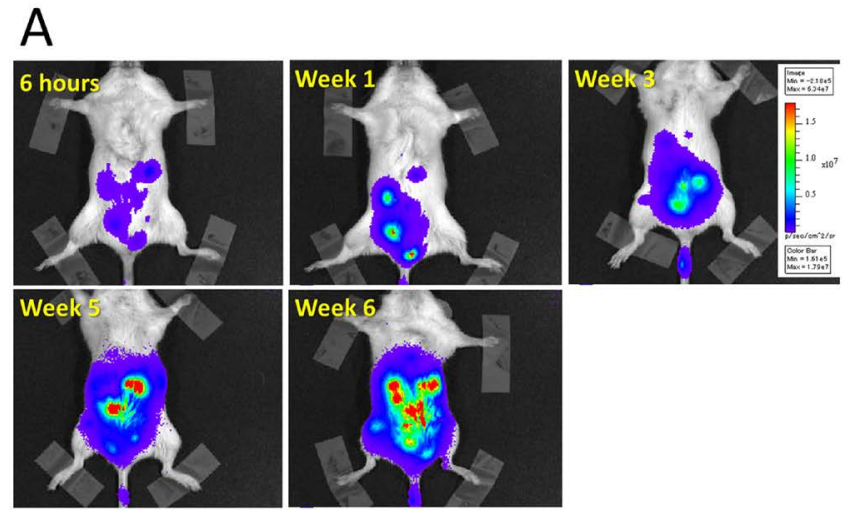

B
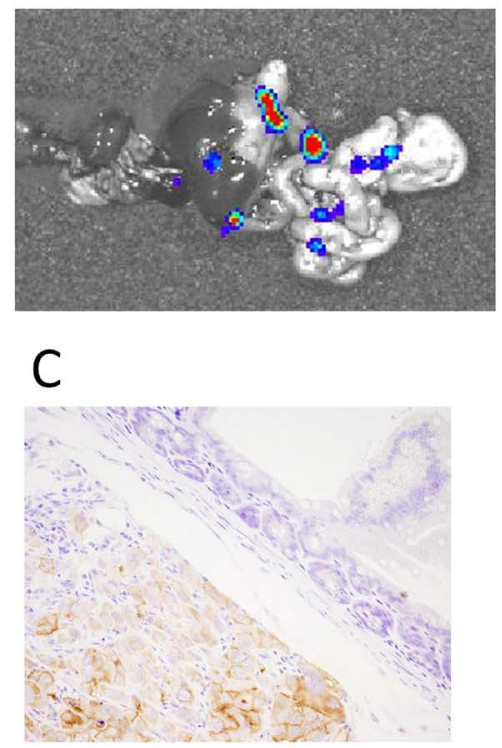

Figure 4: (A) In vivo imaging of peritoneal dissemination model of pancreatic cancer in the NOG mouse showed gradual expansion of abdominal bioluminescent signals throughout the experimental period. (B) Ex vivo imaging revealed the presence of bioluminescence signals in the liver, gastrointestinal tract, and kidneys. (C) The human cancer cell nest formed in the serous surface of intestine was positive for HLA class I-A,B,C. Original magnification, $\times 400$. 
Citation: Yoshimura H, Matsuda Y, Naito Z, Ishiwata T (2013) In Vivo Bioluminescence Imaging of Pancreatic Cancer Xenografts in NOG Mice. J Carcinogene Mutagene S9: 003. doi:10.4172/2157-2518.S9-003

period revealed that the liver was the organ most severely affected with metastatic lesions. The weekly bioluminescence images showed that the metastatic growth process started with a trapping of tumor cells in the lungs after tumor cell inoculation. One week later, most of the tumor cells had died. The surviving cells then began to proliferate and, after 3 weeks, began to form tumors again. Around the same time, new lesions formed in the liver in addition to the lungs. After 5 weeks, the main region of tumor growth shifted from the lungs to the liver. Thus, PANCluc5 cells were first trapped in the lungs, but tumor growth eventually became predominant in the liver, possibly because the liver provides a more suitable microenvironment for PANC-luc5 cell growth.

When mice are injected with luciferin just before sacrificing them, luciferase-expressing tumor cells continued generating light for a short time after dissection. Ex vivo bioluminescence imaging can detect micrometastases in various organs, and even in bones [14]. In the present orthotopic model, no macroscopic metastases were visible in the excised lungs and livers of NOG mice; however, ex vivo imaging detected luminescent signals in the lungs and livers. Microscopic examination revealed small metastatic foci in these organs, verifying that the luminescent signals correctly indicated the presence of tumor cells. These results indicate that ex vivo bioluminescent imaging is useful for preventing missed micrometastases in NOG mice.

In conclusion, in vivo bioluminescence imaging allowed the visualization and quantification of xenograft tumors and their metastases, and of tumors in intravenous and intraperitoneal injection models in the same animal over time. Overall, the implantation of PANC-luc5 cells into NOG mice combined with monitoring by in vivo bioluminescence imaging is a promising model for investigating the mechanisms of pancreatic cancer cell growth and metastasis, and for developing novel anti-cancer drugs for pancreatic cancer.

\section{Acknowledgments}

The authors thank Ms. Yoko Kawamoto, Ms. Kiyoko Kawahara, Ms. Megumi Murase, Ms. Taeko Suzuki, and Mr. Yuji Yanagisawa (Departments of Pathology and Integrative Oncological Pathology), and Dr. Junji Ueda (Department of Surgery, Nippon Medical School) for their excellent technical assistance.

\section{References}

1. Fujii E, Suzuki M, Matsubara K, Watanabe M, Chen YJ, et al. (2008) Establishment and characterization of in vivo human tumor models in the NOD/ SCID/gamma(c)(null) mouse. Pathol Int 58: 559-567.

2. Ito M, Hiramatsu H, Kobayashi K, Suzue K, Kawahata M, et al. (2002) NOD/SCID/gamma(c)(null) mouse: an excellent recipient mouse model for engraftment of human cells. Blood 100: 3175-3182.

3. Watanabe S, Ohta S, Yajima M, Terashima K, Ito M, et al. (2007) Humanized $\mathrm{NOD/SCID/IL2Rgamma(null)} \mathrm{mice} \mathrm{transplanted} \mathrm{with} \mathrm{hematopoietic} \mathrm{stem}$ cells under nonmyeloablative conditions show prolonged life spans and allow detailed analysis of human immunodeficiency virus type 1 pathogenesis. J Virol 81: 13259-13264.

4. Masuda H, Maruyama T, Hiratsu E, Yamane J, Iwanami A, et al. (2007) Noninvasive and real-time assessment of reconstructed functional human endometrium in NOD/SCID/gamma c(null) immunodeficient mice. Proc Nat Acad Sci U S A 104: 1925-1930.

5. Terada $\mathrm{Y}$, Terunuma-Sato $\mathrm{Y}$, Kakoi-Yoshimoto $\mathrm{T}$, Hasegawa $\mathrm{H}$, Ugajin $\mathrm{T}$, e al. (2008) Development of human Graafian follicles following transplantation of human ovarian tissue into NOD/SCID/gammac null mice. Am J Reprod Immunol 60: 534-540.

6. Ninomiya M, Abe A, Yokozawa T, Ozeki K, Yamamoto K, et al. (2006) Establishment of a myeloid leukemia cell line, TRL-01, with MLL-ENL fusion gene. Cancer Genet Cytogenet 169: 1-11.

This article was originally published in a special issue, Pancreatic Ductal Adenocarcinoma handled by Editor(s). Dr. Toshiyuki Ishiwata, Nippon Medical School, Japan
7. Miyakawa Y, Ohnishi Y, Tomisawa M, Monnai M, Kohmura K, et al. (2004) Establishment of a new model of human multiple myeloma using NOD/SCID/ gammac(null) (NOG) mice. Biochem Biophys Res Commun 313: 258-262.

8. Dewan MZ, Watanabe M, Ahmed S, Terashima K, Horiuchi S, et al. (2005) Hodgkin's lymphoma cells are efficiently engrafted and tumor marker CD30 is expressed with constitutive nuclear factor-kappaB activity in unconditioned NOD/SCID/gammac(null) mice. Cancer Sci 96: 466-473.

9. Capellá G, Farré L, Villanueva A, Reyes G, García C, et al. (1999) Orthotopic models of human pancreatic cancer. Ann N Y Acad Sci 880: 103-109.

10. Giubellino A, Woldemichael GM, Sourbier C, Lizak MJ, Powers JF, et al. (2012) Characterization of two mouse models of metastatic pheochromocytoma using bioluminescence imaging. Cancer Lett 316: 46-52.

11. Nishimori H, Yasoshima T, Denno R, Shishido T, Hata F, et al. (2001) A new peritoneal dissemination model established from the human pancreatic cancer cell line. Pancreas 22: 348-356.

12. van der Horst G, van Asten JJ, Figdor A, van den Hoogen $\mathrm{C}$, Cheung $\mathrm{H}$, et al. (2011) Real-time cancer cell tracking by bioluminescence in a preclinical model of human bladder cancer growth and metastasis. Eur Urol 60: 337-343.

13. Au JT, Gonzalez L, Chen CH, Serganova I, Fong Y (2012) Bioluminescence imaging serves as a dynamic marker for guiding and assessing thermal treatment of cancer in a preclinical model. Ann Surg Oncol 19: 3116-3122.

14. Jenkins DE, Hornig YS, Oei Y, Dusich J, Purchio T (2005) Bioluminescent human breast cancer cell lines that permit rapid and sensitive in vivo detection of mammary tumors and multiple metastases in immune deficient mice. Breast Cancer Res 7: R444-454.

15. Scatena CD, Hepner MA, Oei YA, Dusich JM, Yu SF, et al. (2004) Imaging of bioluminescent LNCaP-luc-M6 tumors: a new animal model for the study of metastatic human prostate cancer. Prostate 59: 292-303

16. Zheng J, Xu L, Zhou H, Zhang W, Chen Z (2010) Quantitative analysis of cell tracing by in vivo imaging system. J Huazhong Univ Sci Technolog Med Sci 30: $541-545$

17. Bornmann C, Graeser R, Esser N, Ziroli V, Jantscheff P, et al. (2008) A new liposomal formulation of Gemcitabine is active in an orthotopic mouse model of pancreatic cancer accessible to bioluminescence imaging. Cancer Chemother Pharmacol 61: 395-405.

18. Kidd S, Caldwell L, Dietrich M, Samudio I, Spaeth EL, et al. (2010) Mesenchyma stromal cells alone or expressing interferon-beta suppress pancreatic tumors in vivo, an effect countered by anti-inflammatory treatment. Cytotherapy 12 : 615-625.

19. Lee CJ, Spalding AC, Ben-Josef E, Wang L, Simeone DM (2010) In vivo bioluminescent imaging of irradiated orthotopic pancreatic cancer xenografts in nonobese diabetic-severe combined immunodeficient mice: a novel method for targeting and assaying efficacy of ionizing radiation. Transl Oncol 3: 153-159.

20. Muniz VP, Barnes JM, Paliwal S, Zhang X, Tang X, et al. (2011) The ARF tumor suppressor inhibits tumor cell colonization independent of p53 in a novel mouse model of pancreatic ductal adenocarcinoma metastasis. Mol Cancer Res 9: 867-877.

21. Machida K, Suemizu H, Kawai K, Ishikawa T, Sawada R, et al. (2009) Higher susceptibility of NOG mice to xenotransplanted tumors. J Toxicol Sci 34: 123 127.

22. Ikoma N, Yamazaki H, Abe $Y$, Oida $Y$, Ohnishi $Y$, et al. (2005) S100A4 expression with reduced $\mathrm{E}$-cadherin expression predicts distant metastasis of human malignant melanoma cell lines in the NOD/SCID/gammaCnull (NOG) mouse model. Oncol Rep 14: 633-637.

23. Suemizu H, Monnai M, Ohnishi Y, Ito M, Tamaoki N, et al. (2007) Identification of a key molecular regulator of liver metastasis in human pancreatic carcinoma using a novel quantitative model of metastasis in NOD/SCID/gammacnull (NOG) mice. Int J Oncol 31: 741-751.

24. Dewan MZ, Terunuma H, Ahmed S, Ohba K, Takada M, et al. (2005) Natura killer cells in breast cancer cell growth and metastasis in SCID mice. Biomed Pharmacother 59 Suppl 2: S375-379. 